\title{
NOVEL MINIMAL INVASIVE SURGICAL TECHNIQUES FOR THE TREATMENT OF \\ SEGMENTAL-LATERAL PATHOLOGIC LESIONS OF THE SPINE, PRESERVING THE DORSAL STABILIZING STRUCTURES
}

Zoltán Papp ${ }^{1}$, János Vajda ${ }^{1}$, Róbert Veres ${ }^{1}$, Péter Banczerowski ${ }^{1,2}$ ${ }^{1}$ National Institute of Neurosurgery, Dept. of Spine Surgery, Budapest

${ }^{2}$ Semmelweis University, Faculty of Medicine, Dept. of Neurosurgery zolpapp@oiti.bu

\begin{abstract}
Objective: Laminectomy, the commonly used dorsal approach for the surgical removal of most of the pathologic lesions, situated in the spinal canal and the neuroforamen destroying the dorsal stabilizing structures of the spinal column. Retraction and detachment of the longitudinal muscles, removal of the vertebral arches (laminectomy), and injury of the joint capsules and ligaments are responsible most of short and late-time complications. Among of these complications spinal deformities, segmental instability and permanent spinal pain are most often mentioned in the literature.
\end{abstract}

The main objective of the authors was to develop and evaluate the novel minimally invasive techniques suitable for exploring and treating different segmental-lateral pathologies, expanding to the neuroforamen or even paravertebraly, with preservation of the stability of the spine. One of the key issues of this article was to summarize these procedures, with evaluating the safety and efficacy in the routine spine surgery.

Methods: 153 patients were operated and followed up during 2000 to 2010 in our Institute with segmental lateral spinal pathologies. For the surgical treatment we used "Over-the-top" decompression, hemi-semi laminectomy, supraforaminal burr-hole, open-tunnel and paravertebral approaches alone or combined with each-other. The above mentioned surgical techniques are mostly our developments, or modifications of previously used surgical techniques.

Results: The new surgical procedures developed or modified by our team, are effective techniques for treating the spinal pathologies located in the spinal canal, neuroforamen or even the paravertebral space. The posterior stabilizing structures of the spine, as the vertebral laminae and the longitudinal musculature are mostly preserved. Leaving the longitudinal paraspinal musculature innervations intact, and with the preservation of the bone-muscle attachments and ligaments, the dynamic stability of the spine remains unchanged. Retaining the bony structures (vertebral arches) and the vertebral joints the static stability of the spinal column remain intact, the chance of developing the long-term spinal deformation is minimal.

During the follow up we performed static and dynamic X-ray, MR and CT scans, completed with neurological examinations to evaluate the progression of the illness, the neurological deficit and the actual state of the spinal column. With clinical use and evaluation of the various surgical approaches we determined the main indications and contraindications of the surgical procedures, highlighting the pit-falls and limitations.

Conclusion: The above mentioned minimally invasive surgical approaches are suitable for treating segmental - lateral spinal pathologies. . For the routine clinical use we organized the surgical techniques into a complete framework based on the location of the pathologic lesions. 
Keywords: partial hemilaminectomy, dumbbell tumour, foraminotomy

\section{Introduction}

The use of the classical laminectomy for dorsal approach to the pathologic lesions located in the spinal canal is widely used in spine surgery. Removal even of one vertebral arch, with detachment of the paravertebral muscles and uni- or bilateral destruction of the facet joints and ligaments are leading to the well- known short and long term complications. The most mentioned in the literatures are the persistent pain, spinal deformities, instability, even subluxation. ${ }^{7-8,10,21}$ Several surgical techniques found in the literature to preserve the posterior structures, but most of them focused on multilevel spinal pathologies. ${ }^{4,6,9,11,12,18}$

One spinal segment consists of the two joining vertebras with all of the ligaments and muscles connecting them to each-other. Our novel classification system for treatment of spinal disorders based on the intraspinal localization of pathologic lesions. Mainly, the lesions are localized in the spinal canal in segmental-lateral or axial-longitudinal situation. New minimal invasive spine surgery techniques have been developed with the goal to achieve better clinical outcomes, with the preservation of the dynamic and static stabilizing structures of the spinal column in treatment of the segmental-lateral pathologic lesions. ${ }^{8,9,11-12,17}$

The purpose of our retrospective study is to summarize the minimal invasive procedures with describing technical features, advantages, complications and clinical outcomes for treating segmental-lateral pathologies, located in the spinal canal, in the neuroforamen, or even paravertebraly. $3,5,13,14-16$

\section{Clinical Materials and Methods}

Between 2000 and 2010 the authors performed 153 surgical procedures on patients with segmental-lateral pathologic lesions. Regarding to the minimal invasive surgical concept, every patients were operated with the following surgical techniques, thus are developed by our team, or modification of a previously used surgical technique.

\section{Over-the-top decompression:}

Unilateral approach and short paravertebral muscle retraction performed, throughout a midline incision, on prone positioned patient. To achieve the sufficient decompression of the neural structures the medial part of the articular process, partially the upper and lower laminar arch, the base of spinous process, the ventral part of the interspinosus ligament and the ligamentum flavum on both sides were removed. We operated 51 patients with one segment degenerative spinal canal stenosis.

\section{Hemi-semi laminectomy:}

This approach also mentioned as partial hemilaminectomy. ${ }^{2,22}$ The patient in prone position. The paraspinal muscles are dissected and retracted. The upper and lower arches of the laminae are drilled severally or in case of need altogether at the level of the lesion. The integrity of laminae remains intact. For wider surgical view, the base of spinous process may be removed. The interspinous ligaments are remained intact. 86 patients were operated during the evaluated time 
period (2000-2010) with one to three segment "hemi-semi laminectomy" technique. Histological distribution was as follows: 32 meningeomas, 27 neurinomas, 12 ependymomas, 3 arachnoideal cysts, 4 epidural haematomas, 5, epidural abscesses, 3 dural arterio-venous fistulas. (Figure 1, Figure 2)

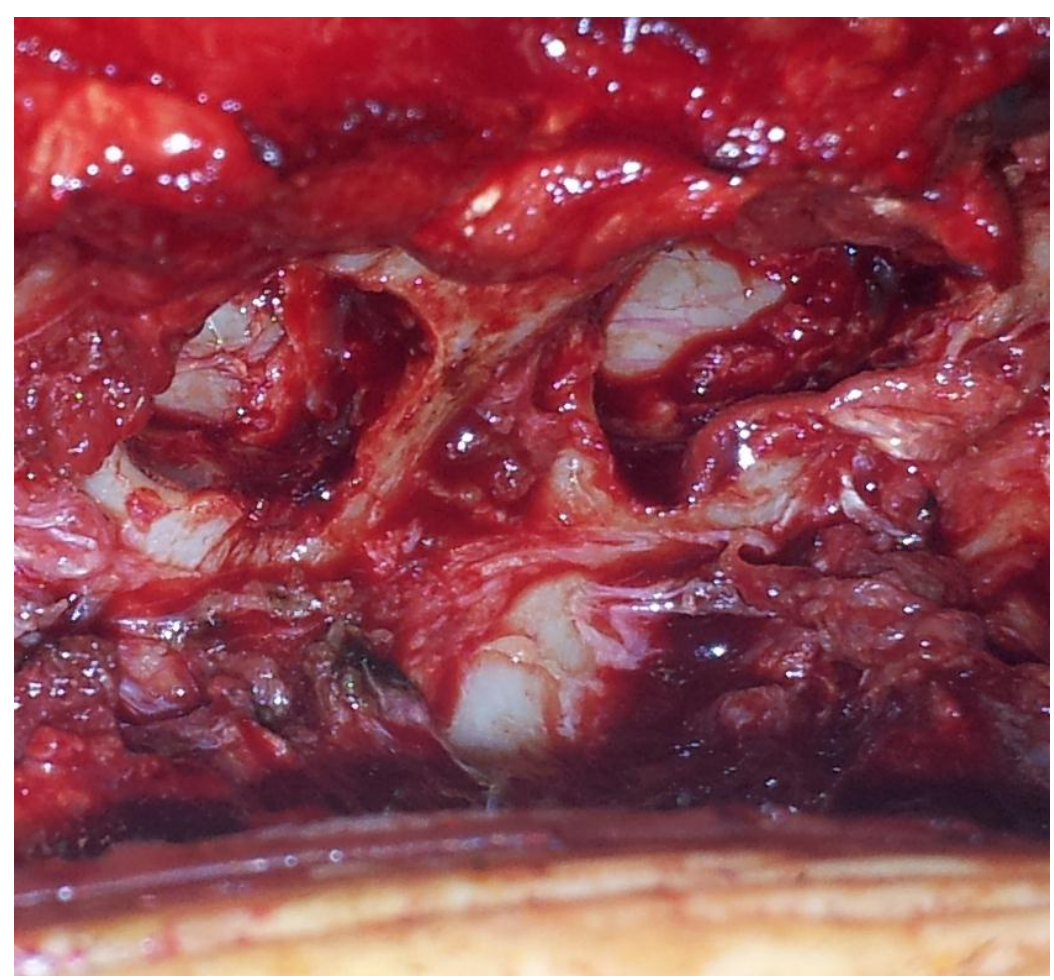

Figure 1. Intraoperative photograph showing two levels hemi-semi approach, on the thoracic spine, right side. The paraspinal muscles retracted on the right side, and the upper and lower arches of the laminae are drilled. The dura is not yet opened.

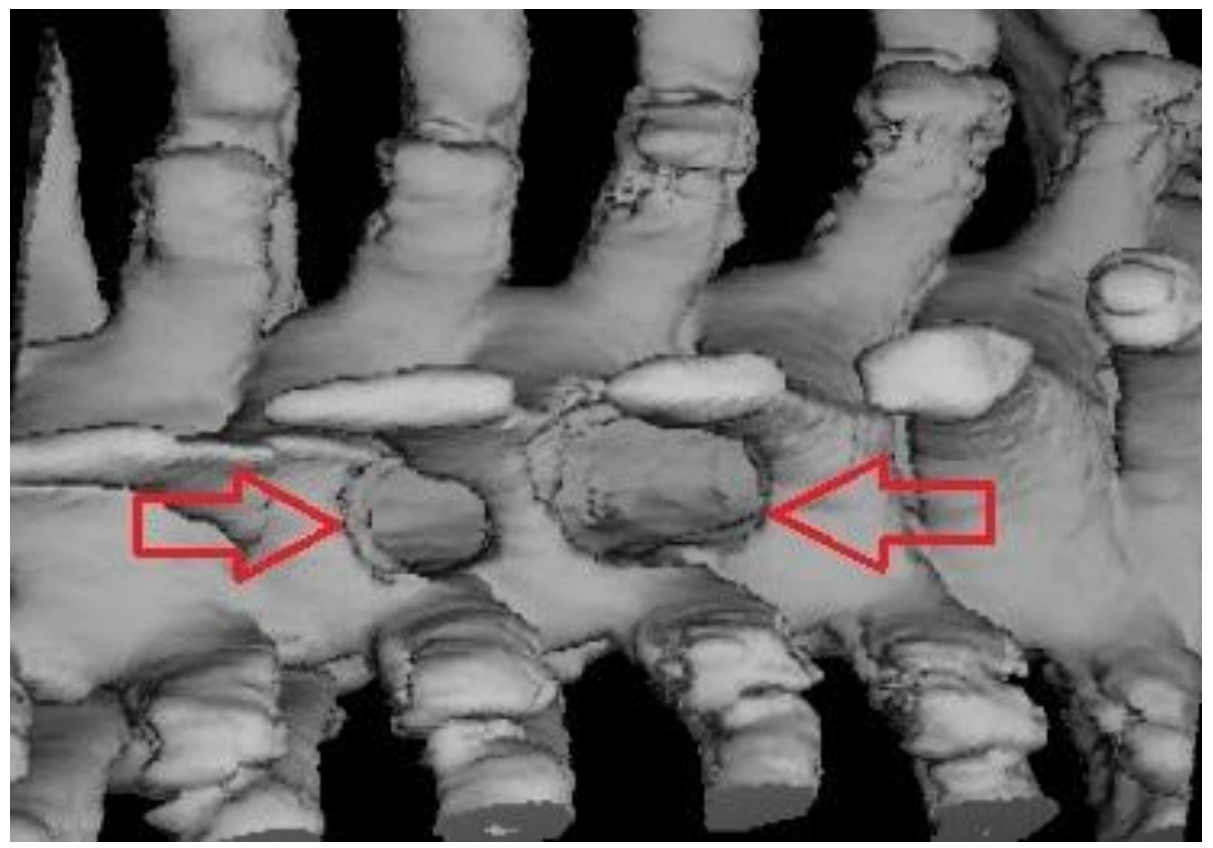

Figure 2. Postoperative three dimensional reconstructed computer tomographic image showing hemi-semi laminectomy approach and the fenestrated laminas on the thoracic spine. 


\section{Supraforaminal burr-hole:}

This approach is suitable for the treatment of lesions extending to the neuroforamen. A hemisemi laminectomy was done the above mentioned way, than the intervertebral foramen exposure was performed across the medial part of the facet joint with a high speed drill with the aim of sparing as much facet as possible. Throughout this $5-7 \mathrm{~mm}$ wide burr-hole the removal of the intraforaminal part of the lesion was achieved. 7 patients were operated with neurinomas extending to the neuroforamen in the cervical spine. In 4 cases we removed schwannomas, and 3 cases neurofibromas. (Figure 3.)

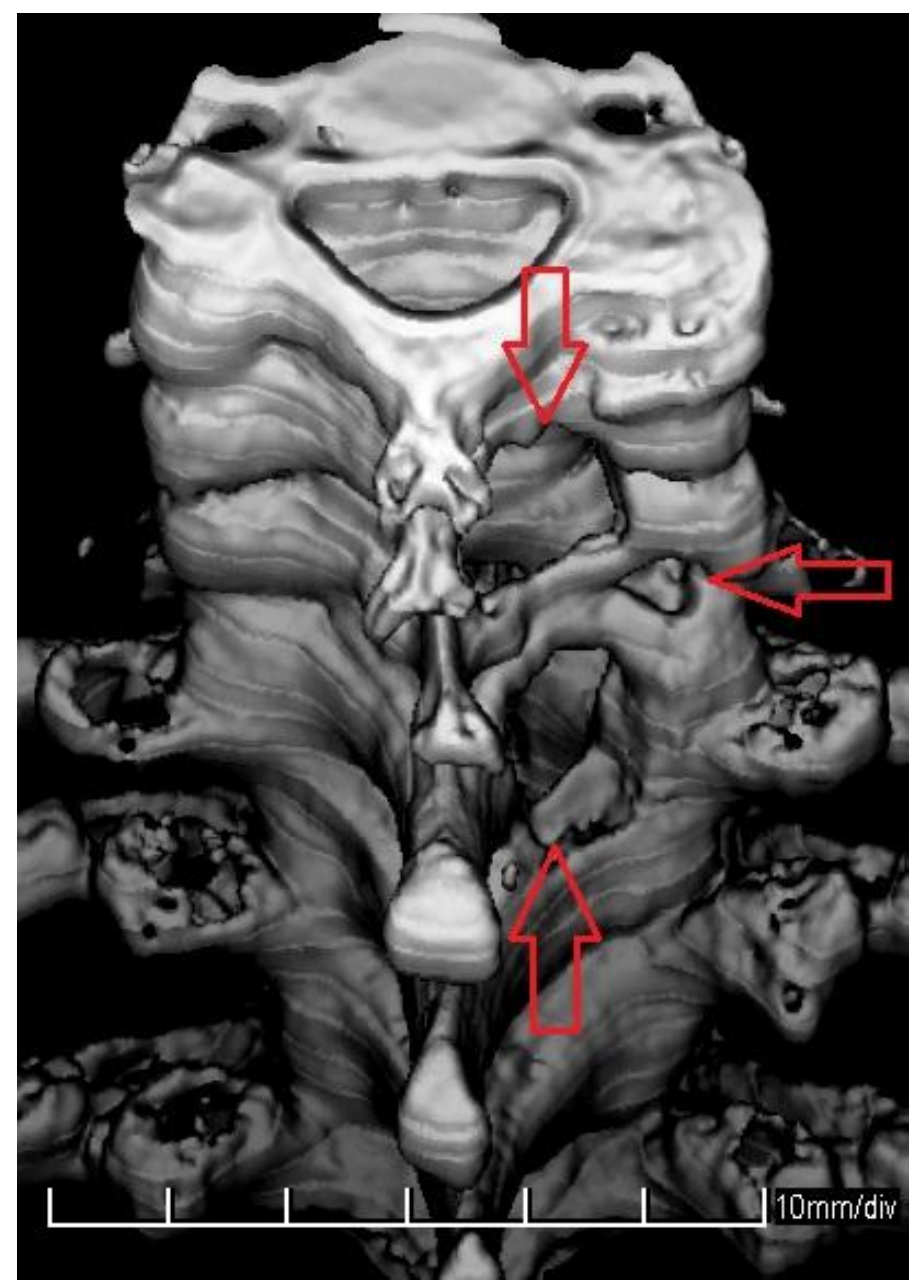

Figure 3. Postoperative three dimensional reconstructed computer tomographic image showing hemi-semi laminectomy with supraforaminal burr-hole approach on the cervical spine.

\section{Open-tunnel:}

This procedure is consists of a hemi-semi laminectomy and the removal of the lateral part of the facet joints. With this approach, both end of the neuroforamen are opened, the tumor removal can be achieved, preserving the bony structures and facet joint as well. Patients are in prone position. After a midline or slightly parasagittal skin incision the paravertebral muscles were dissected and retracted as far as the lateral edge of the facet joints. At the level of the lesion a hemi-semi laminectomy was done. The intraforaminal components of the lesions are exposed from the inner side of the spinal canal through the hemi-semi laminectomy and from the outside made by the partial removal of the lateral part of the facet joint. The exit of the neuroforamen is 
opened and tumor removal is possible from both ends of the opened „tunnel” with sparing the facet joints as much as possible. With this operative technique 6 patients were treated with schwannomas, while 3 with neurofibromas between 2000 and 2010. (Figure 4., Figure 5.)

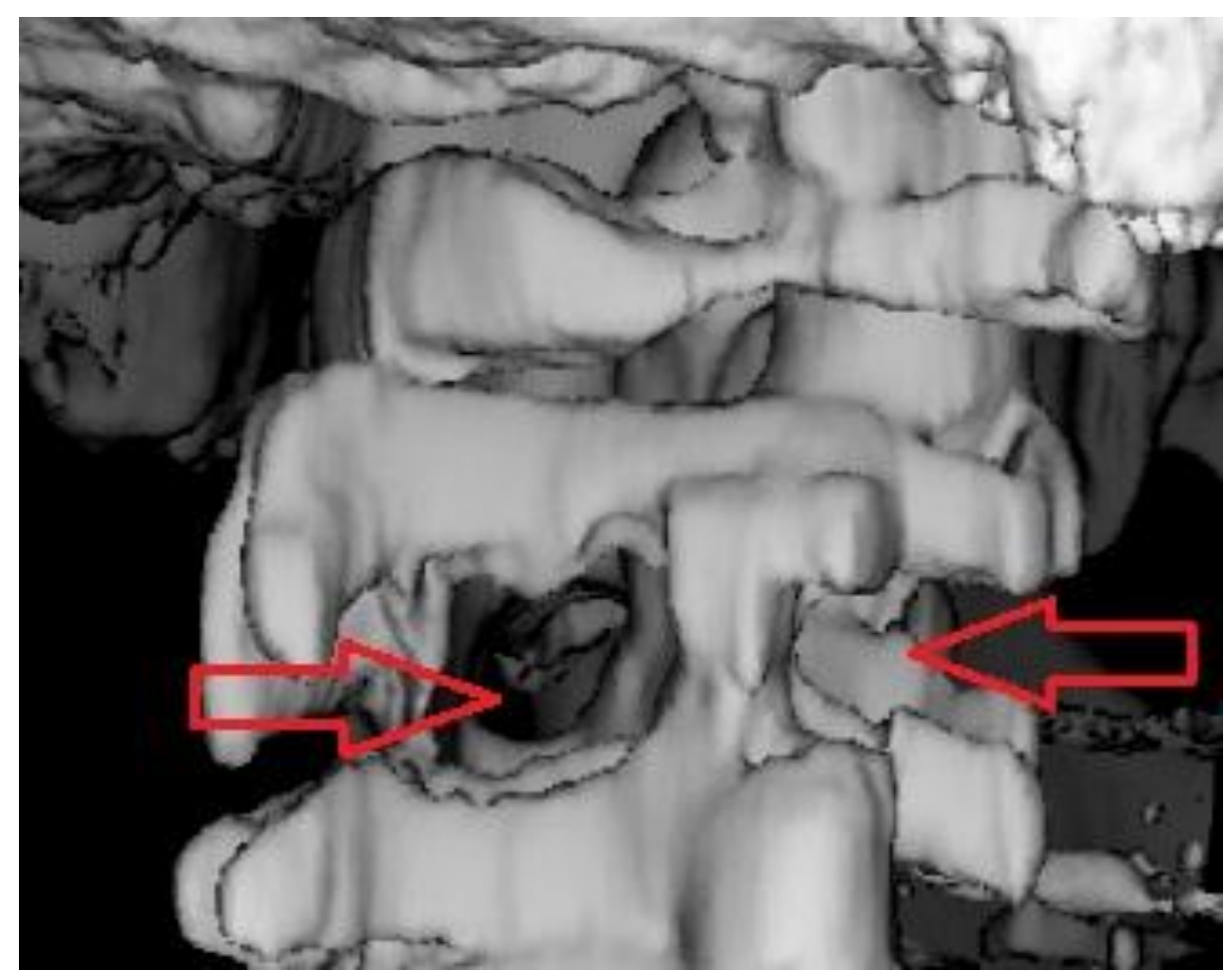

Figure 4. Postoperative three dimensional reconstructed computer tomographic image showing the opentunnel approach at C2 level.

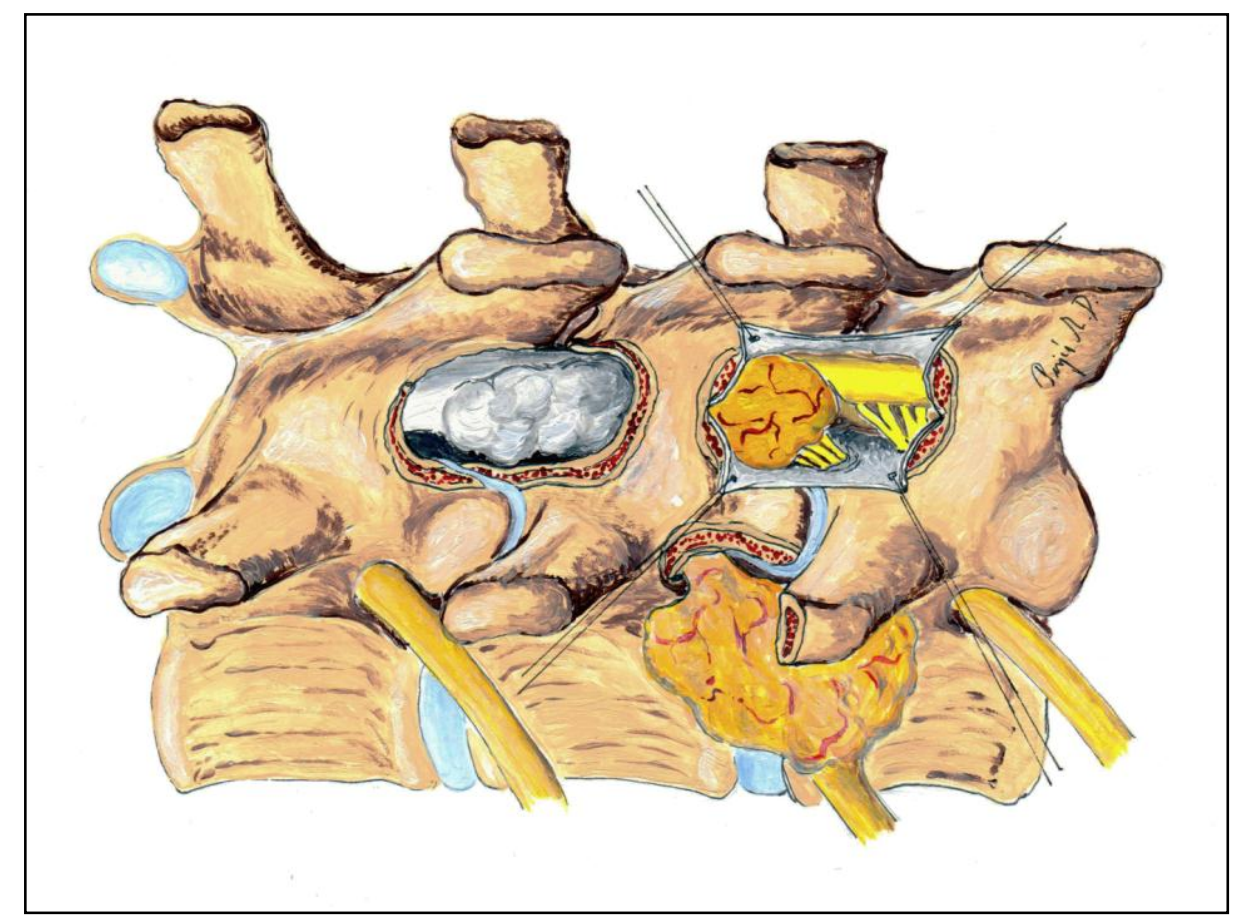

Figure 5. Schematic showing a thoracic dumbbell tumor and the "open-tunnel" approach. The inner part of the tumor removed during two level hemi-semi laminectomy approaches, while the paraspinal part resected directly. The neuroforamen opened at both end, and the remaining tumor was "pulled out". 


\section{Results}

Postoperatively regular control examinations were done. On patients with degenerative spinal canal stenosis we performed CT and MR scans to verify the extent of the decompression of the neural structures. On tumor patients we did regular MR scans to follow the progression of lesion. Both patient group we performed static and dynamic X-ray examinations, to uncover the signs of segmental instability. All of the patients undergone neurological examinations regarding the initial symptoms as well.

All of the surgical approaches and procedures were sufficient to treat the selected pathologic lesions.

$76 \%$ of the patients operated with "over-the-top" decompression technique have neurologic claudication symptoms eliminated, and the remaining $24 \%$ mentioned significant reduction. The low back pain was disappeared in 13\%, reduced in $63 \%$, and not changed in $24 \%$. No sign of instability was detected.

Use of the hemi-semi laminectomy approache all of the pathologic lesions were removed completely.

All of the tumors, expanding to the neuroforamen were removed completely with combination of hemi-semi laminectomy and the supraforaminal burr-hole technique but one, because unsepareable infiltration of the C6 nerve root. Instability in cervical spine flexion and extension did not occur during the follow up period.

The use of "open-tunnel" technique the extent of resection or neurological outcome was satisfactory. Regarding to the minimal invasive surgical approaches the skin incision and paraspinal muscle dissection was shorter, comparing to the "classic" dorsal surgical procedures. Early postoperative mobilization was allowed at every case. The average length of the hospital stay was 7.2 days (range 6-9 days).

No sign of spinal instability found during the follow-up period.

\section{Discussion}

The most frequently used dorsal surgical approach for treatment of spinal lesions has been laminotomy until the last two decades. The surgical treatment of multilevel spinal pathologic lesion requires long, destructive approaches with multiple laminectomies and sacrifice of the attachments of the paraspinal muscles, the facet joints, and the ligaments. To avoid the postoperative complications various types of surgical techniques have been developed. ${ }^{6,9,12,15}$ The main objective of these innovations to preserve and reconstruct the posterior spinal structures. Beyond the disadvantageous effect of the multilevel approaches the preservation of the anatomical and functional spinal segment is highlighted in the literature. For treating the segmental pathologic lesions also the laminectomy was the mostly used dorsal approach. Removal of one laminar arch with cutting the inter,- and supraspinosus ligaments, bilateral detachments of paraspinal muscles and destruction of facet joints are the most frequent cause of disturbance of the sagittal balance. Other important factor for development of long term complications is the segmental instability. The above mentioned irreversible changes accelerate 
the ongoing degenerative procedures on the entire spinal column, advancing the long term symptoms and even neurological impairments.

The literature emphasizes the important role of the deep extensor muscles, especially in the neck. The multifidus and the semispinalis cervicis muscles act as dynamic stabilizers of the cervical spine together with the important static stabilizer structures, including the intervertebral discs, the vertebral arches, ligaments and intervertebral joint capsules. Once these muscles have been detached, it is impossible to reconstruct the complicated anatomy. The integrity of the nerves is also important because if they are injured (branches ramified from the dorsal ramus), preservation of the muscles becomes meaningless. ${ }^{20}$

According to the up-to-date biomechanical concepts new surgical approaches have been developed to avoid the short and long term complications of treatment the (one) segmentallateral pathologic lesions. ${ }^{15,19,20}$ All of these surgical procedures preserving the paraspinal muscle attachments and also the innervation of these muscles. The muscle retraction performed unilateraly, the contralateral side remain totally intact. Using the keyhole concept, the exploration of the spinal canal, and the neuroforamen needs only partial bone removal. The laminar arch remains intact at every approach. In consequence of this the various forces evoking in the course of spinal movements transmitted physiologically throughout the pedicles to the vertebral body. Due to the intervertebral facet joints and ligaments are not injured with these surgical techniques, the segmental stability does not affected.

With the above mentioned methods the operative field is restricted compared to laminectomy, but according to the keyhole principle, it is still enough under the operating microscope for the surgery of lesions located within the spinal canal, in the neuroforamen or paravertebraly.

Between 2000 and 2010 we operated 153 patients with segmental-lateral pathologic lesions with the newly developed or renewed minimal invasive surgical technique. During this period we determined the indications and limits of the procedures and arranged them into a framework based on the localization of the pathologic lesion. Based on our experience, these procedures are safe on all spinal segments (the cervical, thoracic, and lumbar spine) with an acceptable complication rate. Our novel, modified, minimally invasive technique enables surgeons to obtain a sufficient field for exploring different segmental-lateral spinal pathologies that require spinal canal decompression with preservation of the posterior structures of the spine and the attachments of the muscles.

\section{Conclusion}

The minimally invasive surgical techniques for treating segmental-lateral pathologic lesions are safe and effective surgical procedures. The extent of resection are not affected by the newly developed approaches. The posterior dynamic and static stabilizing structures of the spine, as the vertebral laminae, the joint capsules, ligaments, and the longitudinal musculature are mostly preserved. Preservation of these structures helps to avoid the short and long term complications of the widely used laminectomy. 


\section{REFERENCES}

1. Brotchi. Intrinsic spinal cord tumor removal. Neurosurg 2002;50:1059-63.

2. Banczerowski P, Vajda J, Veres R. Removal of intraspinal space-occupying lesions through unilateral partial approach, the "hemi-semi laminectomy ". Clin Neurosci/Ideggy Szle 2008;61:114-22.

3. Cherqui A, Kim DH, Kim SH et al. Surgical approaches to paraspinal nerve sheath tumors. Neurosurg Focus 2007;22:E9

4. Chiou Sh-M, Eggert HR, Laborde G et al. Microsurgical unilateral approaches for spinal tumour surgery: Eight years experience in 256 primary operated patients. Acta Neurochir 1989;100:127-33.

5. Conti P, Pansini G, Mouchaty $\mathrm{H}$ et al. Spinal neurinomas: retrospective analysis and long-term outcome of 179 consecutively operated cases and review of the literature. Surg Neurol 2004;61:34 - 43.

6. Eggert HR, Scheremet R, Seeger W et al. Unilateral microsurgical approaches to extramedullary spinal tumours: Operative techniques and results. Acta Neurochir 1983;67:245-53.

7. Hosono N, Yonenobu K, Ono K. Neck and shoulder pain after laminoplasty. A noticeable complication. Spine 1996;21:1969-73.

8. Hukuda S, Ogata M, Mochizuki T, Shichikawa K. Laminectomy versus laminoplasty for cervical myelopathy: brief report. J Bone Joint Surg (Br) 1988;70:325-6.

9. Kato Y, Kaneko K, Kataoka H et al. Cervical hemilaminoplasty: technical note. J Spinal Disord Tech 2007;20:296-301.

10. Katsumi Y, Honma T, Nakamura T. Analysis of cervical instability resulting from laminectomies for removal of spinal cord tumour. Spine 1989;14:1171-6.

11. Kehrli P, Bergamaschi R, Maitrot D. Open-door laminoplasty in pediatric spinal neurosurgery. Child Nerv Syst 1996;12:551-2.

12. Koch-Wiewrodt D, Wagner W, Perneczky A. Unilateral multilevel interlaminar fenestration instead of laminectomy or hemilaminectomy: an alternative surgical approach to intraspinal space-occupying lesions. J Neurosurg Spine 2007;6:485-92.

13. Lot G, George B. Cervical neuromas with extradural components: surgical management in a series of 57 patients. Neurosurgery 1997;41:813-20.

14. MacCormick PC. Surgical management of dumbbell tumors of the cervical spine. Neurosurg 1996;38 :294-300.

15. Ogdena AT, Bresnahanb L, Smith JS, Natarajand R, Fesslerb RG. Biomechanical comparisons of traditional and minimally invasive intradural tumor exposures using finite element analysis. Clinical Biomechanics 2009;24:143-7.

16. Ozawa H, Kokubun S, Aizawa T et al. Spinal dumbbell tumors: an analysis of a series of 118 cases. J Neurosurg Spine 2007;7:587-93.

17. Sarioglu AC, Hanci M, Bozkus H et al. Unilateral hemilaminectomy for the removal of the spinal space-occupying lesions. Minim Invas Neurosurg 1997;40 :74 -7.

18. Shikata J, Yamamuro T, Shimizu K et al. Combined laminoplasty and posterolateral fusion for spinal canal surgery in children and adolescents .Clin Orthop Related Res 1990;259:92-9.

19. Raimondi AJ, Guiterrez FA, Di Rocco C. Laminotomy and total reconstruction of the posterior arch for spinal canal surgery in childhood. J Neurosurg 1976;45:550-60.

20.Zhang J, Tsuzuki N, Hirabayashi S, Saiki K, Fujita K: Surgical anatomy of the nerves and muscles in the posterior cervical spine: A guide for avoiding inadvertent nerve injuries during the posterior approach. Spine 2003;28:1379-84.

21. Yasuoka S, Peterson HA, MacCarthy CS. Incidence of spinal column deformity after multilevel laminectomy in children and adults. J Neurosurg 1982;57:441-5. 
22. Yasargil MG, Tranmer BI, Adamson TE et al. Unilateral partial hemilaminectomy for the removal extra- and intramedullary tumors and AVMs. In: Symon L, ed. Advances and technical standards in neurosurgery. Vol 18, Springer Verlag, Wien; 1991. p.113-32. 